\title{
The case for the Higgs boson
}

\author{
Mr William Waldegrave, Britain's cabinet minister in charge of science, has offered a bottle of decent champagne \\ to one who can tell him why the Higgs boson is worth finding (see page 781). These notes may be helpful.
}

Do not mention gauge invariance, nonAbelian or otherwise. Nor should you begin with a reference to spontaneous symmetry breaking. Of course, there are now plenty of neat proofs that the ground state of a quantum system does not necessarily enjoy the full symmetry of the system as a whole. The obvious case is that of a ferromagnet cooled below the Curie temperature, when the rotational symmetry of the system may be lost by magnetization.

Waldegrave is both inquisitive and intelligent. That he takes his job seriously is borne out by his willingness to worry about the Higgs boson. Evidently the question has arisen in discussions over what should happen next at the European Laboratory for Particle Physics (CERN). With a British director (Dr Christopher LlewellynSmith) now at Geneva, Waldegrave cannot just walk away from the plan to install in the LEP tunnel the Large Hadron Collider, part of whose promise is success in the hunt for the Higgs boson. It is uncharacteristically diligent of a minister to seek precisely to understand what money spent will accomplish. But for all those virtues, it would be ridiculous to expect that he would be enlightened by an account of gauge invariance or symmetry-breaking. Avoid both concepts.

Analogy is the only way to start, and the electron and the electromagnetic field the only place. That approach has also the benefit of introducing several British names of which ministers other than Waldegrave will have heard. (Faraday is now on the $£ 20$ banknote.) So begin along these lines: With respect, Minister, it's to do with explaining action at a distance. Newton worried about that when his theory of gravitation required apparently instantaneous interaction between two distant objects. By Faraday's time, the problem was more acute; electrically charged objects were known to repel or attract each other, as did magnetic poles. Faraday began talking of magnetic fields (and you'll remember the school experiments with iron filings around bar magnets). Later, Faraday showed that moving charges made magnetic fields and so we had the electromagnetic field. By the time of Maxwell, it was clear that the electromagnetic field was the means by which electrical charges and magnetic dipoles act on each other 'at a distance'

Having begun in this historical vein, you had better stay with it. If you consider that the recitation of the names of British heroes will advance your cause, you might even interpolate:

To begin with, people thought that electric charges could be arbitrarily big or small, but Faraday made the crucial step of showing that charged atoms, or ions, are always associated with a fixed amount of charge or some multiple thereof, suggesting atoms of electric charge. Then $J$. J. Thomson showed that these atoms of charge exist; we call them electrons. So far as we know, there are no corresponding atoms of magnetic charge.

It would be unwise to elaborate on the last point. This is hardly the place for a dissertation on magnetic monopoles; indeed, with luck, you can hope to avoid a single mention of the Grand Universal Theories (called GUTs) that predict them. But you cannot avoid explaining how the coming of relativity and then of quantum mechanics influenced thinking on the electromagnetic field. Try something like this: Relativity fitted naturally with Maxwell's electromagnetism, explaining for example why the electromagnetic field around a charge apparently at rest is purely electrostatic, but that around a moving charge (or an electric current) has magnetic components as well. (Mention of $\mathrm{H}$. A. Lorentz at this point would be courteous, Hollander though he may have been.) But the coming of quantum mechanics was the very devil. For a decade until the late 1930s, people could do no better than to regard the electron as an empirical fact. Only after Dirac had predicted that there must be positrons as well as electrons (verified in 1932) did he and others set out to see whether the charge and the mass of an electron can be derived from first principles. He did not get far.

Now comes the difficult part. The problem is how to explain the quantization of the electromagnetic field to one who cannot instinctively write down Maxwell's equations (which is no shame), let alone say what they mean. Hand-waving is unavoidable. A few references to the successes of quantum mechanics in the $1930 \mathrm{~s}$ might be appropriate; in retrospect, Heitler's quantum theory of radiation is especially prescient. But you may be running out of space. So try this:

Dirac was defeated by the complexity of the problem. The quantum theory of an atom requires that each of its components, its electrons for example, should be dealt with separately, but at worst there is only a finite number of them. But the electromagnetic field has an infinite number of variables, one for each point in spacetime. Finishing Dirac's problem was everybody's goal in the 1940s. Feynman, Schwinger and Tomanaga won the prize. The upshot was a picture in which electrons interact by exchanging photons with each other. But they are not real photons at all, but virtual photons created out of the vacuum.

There's now a dilemma. Do not raise awkward questions such as whether quantum electrodynamics, which calculates an electron mass by subtracting infinities by the technique of renormalization, attains Dirac's goal of deriving the properties of the electron from first principles. Most think it does not. In any case, you've almost used up all your space without mentioning the Higgs boson: best do so quickly: The Higgs boson, Minister, plays much the same role as the photon, but in a different context. Rutherford's school saw the difference between $\alpha$ and $\beta$ radioactivity. The second kind, in which nuclear particles give off electrons, forms a bridge between nuclear matter (neutrons for example) and non-nuclear matter such as electrons. In other words, nuclear and non-nuclear matter act on each other. It's action at a distance all over again. So there's another field filling the whole of space that must be quantized. What that exercise predicts is that there should be particles mediating the influence of that field just as the photon mediates the electromagnetic field. They've been found, at CERN. And then there's the last of them, the Higgs boson. That's why its discovery is the last goal for the time being of particle physics.

That, sadly, is all you have room for on one side of a sheet of A4 paper. Do not be downcast that you have been economical with the truth. Not to have mentioned the strong interaction and the quarks and gluons thereby made necessary is barely forgivable, the omission of neutrinos and the lepton generations other than the electron (the $\mu$ and $\tau$ particles) is less so.

You may also cringe a little at your conclusion, knowing as you do that the correctness of the electro-weak theory has been essentially verified by the observation of neutral-current processes as well as by the discovery of the $\mathrm{W}^{ \pm}$and $\mathrm{Z}^{0}$ particles. The real interest of the hunt for the Higgs particle is that it may not be quite what is expected. But best not say that too loudly. 